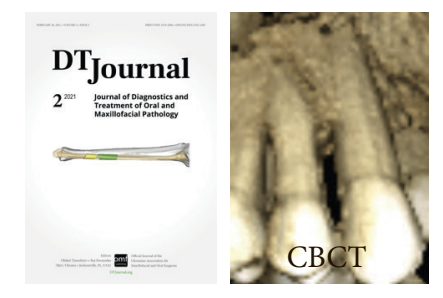

\title{
Prevention of Inflammatory Complications in Fractures of Alveolar Processes of the Jaw
}

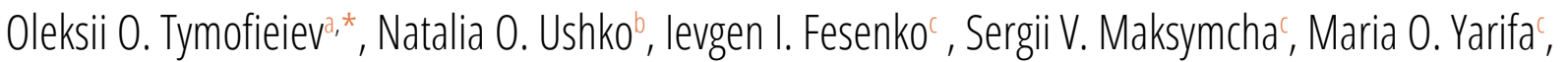 \\ Viktoriia M. Ripa , Anton 0. Myroshnyk, Olexander 0. Savytskyi', Sergii l. Dubichenko, Viktoriia P. Blinovae, \\ Oksana A. Uharska, Olena 0. Serga
}

\section{SUMMARY}

Purpose: To determine the effectiveness of the nonsteroidal anti-inflammatory drug "Tantum Verde" in patients with fractures of the alveolar processes of the maxilla and mandible, to evaluate its effectiveness for the prevention of inflammatory complications.

Methods: Clinical and laboratory examination of 129 patients with fractures of the alveolar processes of the jaws.

Results: Based on the results of the patients examination with open fractures of the jaws. It was found that the analgesic, anti-inflammatory and deodorant efficacy of the nonsteroidal anti-inflammatory drug "Tantum Verde" is significantly higher than traditional therapy, and also has a smaller number of inflammatory complications.

Conclusions: The use of the nonsteroidal anti-inflammatory drug "Tantum Verde" made it possible to significantly reduce the number of inflammatory complications and reduce the treatment duration of patients. It was established that the drug "Tantum Verde" is an effective analgesic and anti-inflammatory drug and can be recommended for the treatment of patients with fractures of the alveolar processes of the jaws.

\footnotetext{
Kyiv, Ukraine

a ScD, Professor, Head, Department of Maxillofacial Surgery, Shupyk National Healthcare University of Ukraine.

${ }^{b} \mathrm{ScD}$, Professor, Department of Maxillofacial Surgery, Shupyk National Healthcare University of Ukraine.

c PhD, Associate Professor, Department of Oral and Maxillofacial Surgery, Private Higher Educational Establishment "Kyiv Medical University."

d Senior Laboratory Assistant, Department of Oral and Maxillofacial Surgery, Private Higher Educational Establishment "Kyiv Medical University."

e Assistant Professor, Department of Oral and Maxillofacial Surgery, Private Higher Educational Establishment "Kyiv Medical University."

* Corresponding author's address: Department of Maxillofacial Surgery, Shupyk National Healthcare University of Ukraine, 10A Pymonenko Street, Kyiv 04214, Ukraine.

E-mail: tymofeev@gmail.com (Oleksii Tymofieiev)

ORCID: https://orcid.org/0000-0002-3191-6025
}

\author{
Please cite this article as: Tymofieiev OO, Ushko NO, Fesenko II, \\ Maksymcha SV, Yarifa MO, Ripa VM, Myroshnyk AO, Savytskyi OO, \\ Dubichenko SI, Blinova VP, Uharska OA, Serga OO. Prevention of \\ inflammatory complications in fractures of alveolar processes of the jaw. $J$ \\ Diagn Treat Oral Maxillofac Pathol 2021;5(2):20-31. \\ The word ' $\mathrm{CBCT}$ ' at the upper right icon means that article contains cone- \\ beam computed tomography scans. \\ Paper received 15 December 2020 \\ Accepted 7 January 2021 \\ Available online 28 February 2021 \\ https://dx.doi.org/10.23999/j.dtomp.2021.2.2 \\ (c) 2021 OMF Publishing, LLC. This is an open access article under the CC
} BY license (http://creativecommons.org/licenses/by-nc/4.0/). 


\section{INTRODUCTION}

Fractures of the alveolar processes of the jaws (Fig 1) occupy one of the leading places among the fractures of the facial skeleton bones. ${ }^{1-5}$ These fractures of the jaw bones include the injury of the alveolar processes and mucous membranes, thus they are associated with the oral cavity and are always infected. Fractures of the alveolar processes of the maxilla and the mandible occur at any age, more often in adolescence. ${ }^{2}$ The most common method of reposition and fixation of jaw bone fragments in fractures is the use of wire or arch bars, which are attached to the teeth with a wire. All these dental metal structures (arch bars, wires) are located on the oral cavity vestibule. It should be noted that any design of dental splints used to fix bone fragments makes it impossible to eat normally.
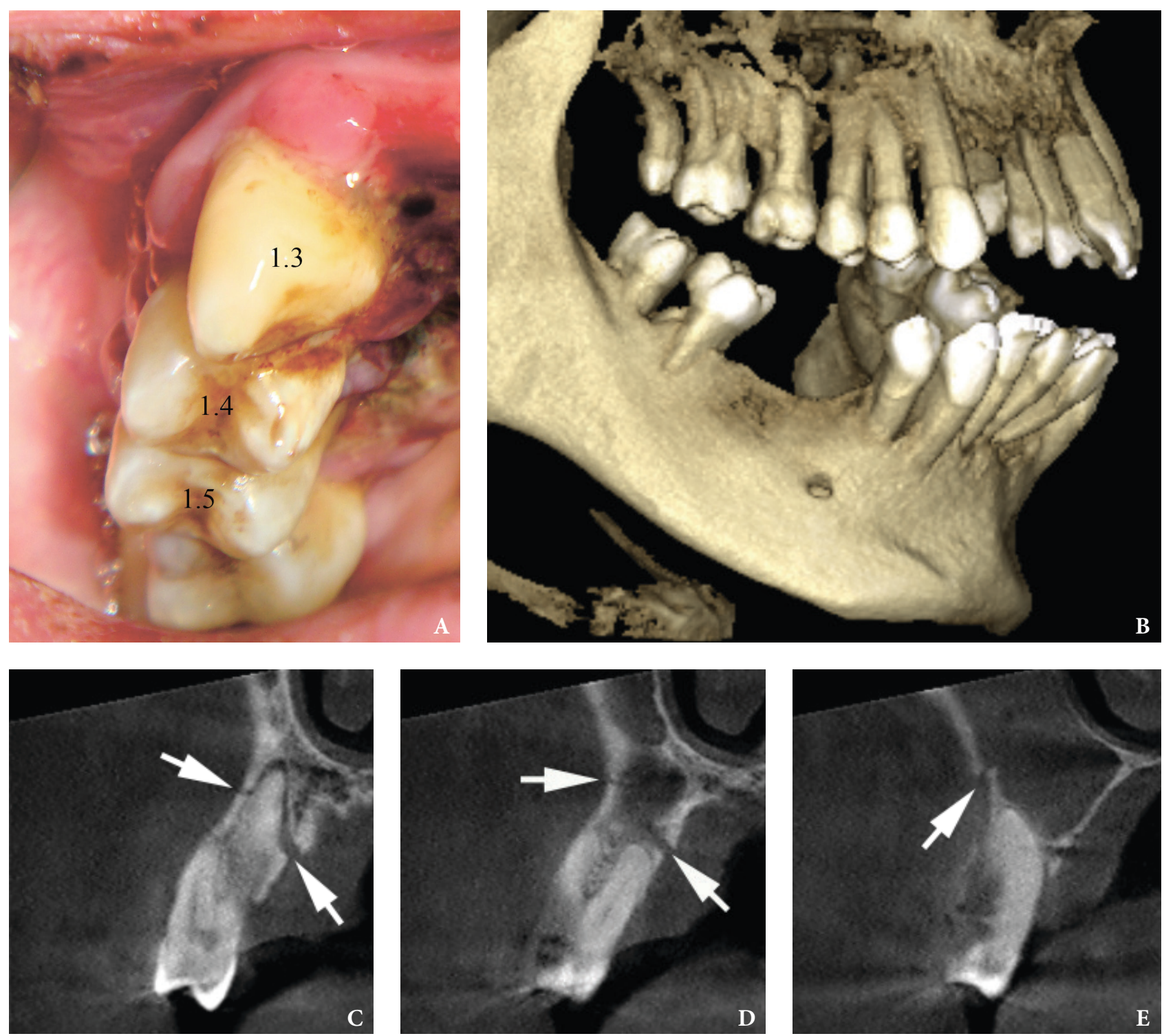

FIGURE 1. A 45-year-old Caucasian male after the injury. Intraoral view $(\mathbf{A})$ shows mucosal laceration and buccal dislocation of the teeth 1.5, 1.4, and 1.3. Cone-beam computed tomography (3-dimensional $[\mathbf{B}]$ and coronal scans $[\mathbf{C}-\mathbf{E}]$ ) shows segmental alveolar fracture (arrows) at the right maxilla with dislocation of involved teeth 1.5-1.3. Image $\mathbf{C}$ demonstrates tooth 1.3 and fracture line/gap located coronal to apical foramen. Image $\mathbf{D}$ visualize tooth 1.4 and fracture line located apical to apical foramen. Image $\mathbf{E}$ shows tooth 1.5 and fracture line located apical to apical foramen. Printed with permission and copyrights retained by I.I.F. 
It is very difficult to clean the metal structures in the patient's mouth. Many additional retention points appear in the oral cavity, where food debris can be retained, which are a medium for the development of pathogenic microflora. Oral hygiene is of great importance for the prevention of inflammatory complications in the treatment of patients with fractures of the alveolar processes of the maxilla and the mandible. Individual oral hygiene of patients with jaw fractures contributes not only to the removal of food debris and soft dental plaque, which are located on the splinting metal structure, ligature wire, teeth, gingiva, but is also a prophylaxis for the development of microbial flora of the oral cavity vestibule. The absence of food debris on the dental metal structures and the absence of pathogenic microflora make it possible for a faster and more favorable healing of the bone fragments of the jaws.

Thus, hygiene measures for the oral cavity care of patients with fractures of the alveolar processes of the jaw bones is one of the important factors for the prevention of complications such as gingivitis and the development of a purulent-inflammatory process in the fracture gap of injured bones (post-traumatic osteomyelitis).

The patient regularly undergoes not only medical oral hygiene, i.e. the doctor teaches the patient to carry out hygiene measures for the oral cavity care and exercises control. The patient must independently carry out individual hygiene care of the metal structures in his oral cavity. The traditional method of medical oral hygiene for fractures of the jaws is irrigation (using a stream of antiseptic from a syringe) of additional retention points with solutions of hydrogen peroxide, potassium permanganate (pale pink solution), chlorhexidine bigluconate, furacilin, etc. With the help of a toothbrush, the patient cleans metal arch bars, wires and teeth from food debris, and then antiseptic irrigation and rinsing of the oral cavity vestibule are repeated. Individual hygiene should be carried out by the patient not only after each meal, but also in the intervals between meals, as well as before bedtime.

When choosing an antimicrobial drug for hygienic oral care, you need to focus on the preventive purpose of its use, ie to prevent the development of inflammation from the mucous membranes of the oral cavity. For these purposes, the patients use a toothbrush to clean arch bars, wires and teeth from food debris, and then carry out antiseptic irrigation and antiseptic rinsing of the oral cavity with a nonsteroidal anti-inflammatory drug "Tantum Verde" approved for use in Ukraine (order of the Ministry of Health of Ukraine No. 1789 dated August 04, 2020; Registration certificate UA / 3920/02/01). ${ }^{6}$

The drug "Tantum Verde" is available in the form of a solution in a package of $120 \mathrm{ml}$ (Fig 2). Tantum Verde is a 0.15 percent solution for local application in the form of a clear green liquid with a characteristic mint odor. $1 \mathrm{ml}$ of solution contains benzydamine hydrochloride $1.5 \mathrm{mg}$; excipients: ethanol 96 percent, glycerin, methyl p-hydroxybenzoate (E218), flavor enhancer (menthol), saccharin, sodium bicarbonate, polysorbate 20, quinoline yellow 70 percent (E104), patented blue V 85 percent (E131), purified water. The active substance of the drug - benzydamine, is a non-steroidal anti-inflammatory drug (NSAID), which has an expressed anti-exudative and analgesic effect. When applied topically, benzydamine acts as a disinfectant. Its effectiveness when applied topically is due to its ability to penetrate the epithelial layer and reach effective concentrations in inflamed tissues. The mechanism of action of benzydamine is associated with the stabilization of cell membranes and inhibition of prostaglandin synthesis. The antibacterial activity of the active substance is manifested due to the rapid penetration of microorganisms through the outer membranes, followed by damage to cellular structures, disruption of metabolic processes and cell lysis. Benzydamine restores the integrity of the mucous membranes epithelium, increases its resistance to pathogenic effects. When applied topically in the indicated concentrations, benzydamine is absorbed by the mucous membrane, but its concentration in the blood plasma is so low that it cannot cause any pharmacological effect. Benzydamine is excreted in the urine in the form of inactive metabolites or conjugation products.

Contraindications are: hypersensitivity to the drug, pregnancy and lactation. Benzydamine is not recommended for patients with hypersensitivity to salicylic acid or other NSAIDs. When using the drug, sometimes there is a feeling of numbness or burning at the area of application, which is associated with the presence of ethanol in the drug. In some cases, allergic reactions may occur - skin rash, dry 


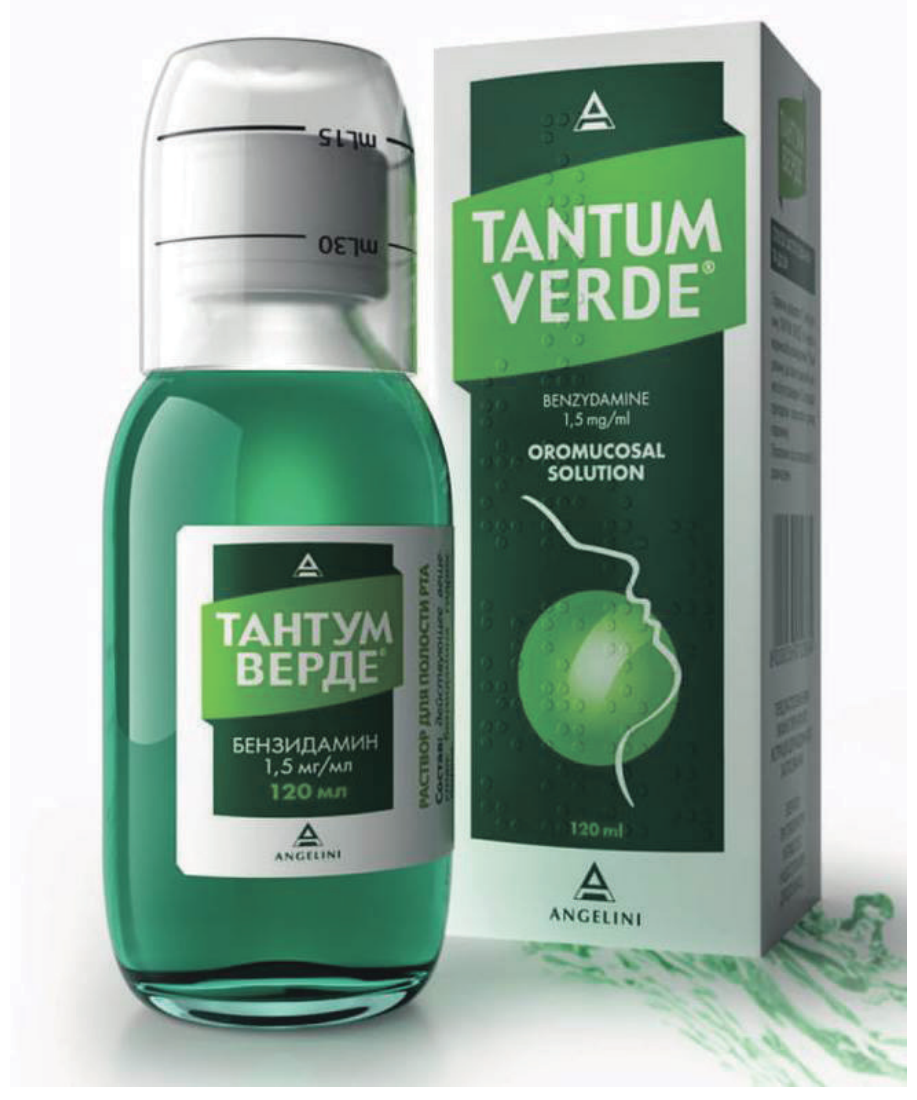

FIGURE 2. Appearance of the "Tantum Verde."

mouth, bronchospasm, angioedema or other allergic reactions, as well as swelling and discoloration of the tongue, change in taste. For athletes: the use of medicines containing ethyl alcohol can give a positive result under doping control. When using the drug in the recommended doses, we did not observe any side effects. In case of an overdose of the drug, dry mouth, drowsiness, and allergic reactions are possible. Tantum Verde can be used by children over 12-year age.

Method of application of the drug "Tantum Verde": for rinsing the mouth, we used $15 \mathrm{ml}$ (1 tablespoon or a measuring cup from a bottle) of Tantum Verde (can be diluted in $15 \mathrm{ml}$ of water). Rinsing was performed 5-6 times a day. After rinsing, spit out the solution. You can't swallow it.

The aim of the study is to determine the effectiveness of the use of the drug "Tantum Verde" for the prevention of inflammatory complications in the oral cavity of patients with injuries (fractures) of the alveolar processes of the maxillary and mandibular bones.

\section{MATERIAL AND METHODS}

A total of 129 patients with fractures of the alveolar processes of the maxilla and the mandible were examined. The main group consisted of 89 patients with fractures of the alveolar processes of the maxilla and the mandible at the age from 13 to 43 years. The drug "Tantum Verde" was prescribed by us in the form of antiseptic rinses. For these purposes, a measuring cap was used, 15 $\mathrm{ml}$ of the Tantum Verde solution was measured and undiluted or diluted $(15 \mathrm{ml}$ of the solution can be diluted with $15 \mathrm{ml}$ of water) rinsed the oral cavity. Rinsing was carried out 3 times a day. The course of treatment was 10-12 days. General drug treatment was not prescribed to the patients. The control group consisted of 40 patients with the same injuries and the same age who, in the dynamics of the treatment, used a solution of chlorhexidine digluconate ( 0.05 percent) for antiseptic baths (Fig 3). The patients were not prescribed general drug treatment. 


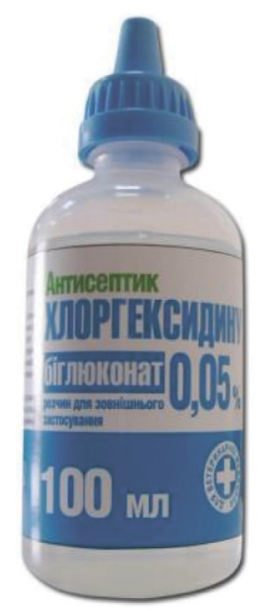

FIGURE 3. 0.05\% chlorhexidine digluconate appearance.

In the dynamics of examination and treatment of patients, a general clinical examination was carried out, which included: clarification of complaints, pain examination, palpation, anamnesis, X-ray/computed tomography of the jaws, general blood test.

In the dynamics of the examination, microbiological methods were carried out (microflora and its antibiotic sensitivity were determined). Material from the tooth-gingival pockets in the area of the fracture gap (rupture of the mucous membrane of the alveolar process) was taken both by the standard method (using a sterile cotton swab) and using a Volkmann spoon (to identify common microflora and fungi). Microbiological studies were performed in the morning on an empty stomach. The collected material was carefully applied onto a sterile glass slide (the material should not be rubbed on the glass, as this may damage the delicate elements of the fungus). Microscopic examination of unstained and also Romanovsky-Giemsa stained native preparation was performed. The sowing of the discharge from the tooth-gingival pocket was transferred to the Sabouraud nutrient medium, followed by sowing on special medium and identification of the pathogenic agent.

Evaluation of the effectiveness of the use of hygiene products was determined by the following indices. To detect the presence of an inflammatory process of the alveolar processes mucous membrane, the SchillerPisarev test was performed. The mucous membrane of the alveolar processes was treated with Lugol's solution. The digital value of the Schiller-Pisarev test (Svrakov iodine number) was determined in points.
Evaluation of the Svrakov iodine number values:

- weakly expressed process of inflammation - up to 2.3 points;

- moderately expressed inflammation process 2.67-5.0 points;

- intense process of inflammation - 5.33-8.0 points.

Gingivitis index was proposed in 1967 by SilnessLoe. Evaluated on a 4-point system:

0 - no inflammation;

1 - mild inflammation (slight discoloration);

2 - moderate inflammation (hyperemia, edema, possible hypertrophy);

3 - severe inflammation (severe hyperemia).

Index evaluation criteria:

0.1-1.0 - mild degree of gingivitis;

1.1-2.0 - average degree of gingivitis;

2.1-3.0 - severe degree of gingivitis.

Contact thermometry was carried out using a TPEM-1 electrothermometer with a resolution of $0.2^{\circ} \mathrm{C}$. Contact thermometry is based not on the measurement of absolute temperatures over the pathological focus, but on the identification of the temperature difference in symmetrical areas $(\Delta \mathrm{T})$. Thermal asymmetry $(\Delta \mathrm{T})$ in symmetrical areas, revealed in practically healthy people of the same age and sex, served as a control. The temperature of the 
mucous membrane of the alveolar processes of the upper and lower jaws of the patients was measured in the area of bone injury (the area of the fracture gap) and on the symmetrical (intact) healthy side. The contact temperature was measured at the first visit of the patient, as well as in the dynamics of the treatment.

Clinical symptoms and the obtained digital data of laboratory examinations were processed by the variation-statistical method using a personal computer. The reliability of the results was calculated according to Student's $t$-test. Differences were considered significant at $p<0.05$.

\section{RESULTS AND DISCUSSION}

Microbiological examinations were carried out in 80 patients of the main group, and in the control group of observation - in 38 people.

In all 80 patients of the main group and 38 patients in the control observation group, microorganisms were sown on the 3-4th day after the reposition of the jaw fragments, ie 100 percent. Material for microbiological studies was taken from the tooth-gingival pockets in the area of the fracture gap (rupture of the mucous membrane of the alveolar process).
In the main observation group, upon treatment of patients, Staphylococcus aureus was sown from the periodontal pockets (Fig 4) in 77.5 percent (in 62 of 80 people), Staphylococcus epidermidis - in 53.8 percent (in 43 of 80 people) and hemolytic Streptococcus - in 27.5 percent ( 22 out of 80 people). In 20 of 80 patients of the main group (in 25 percent), the causative agent of Candida: Candida albicans and Candida tropicalis were found in the tooth-gingival pocket. Of the 80 patients in the main group, 52 patients ( 65 percent) were sown with monocultures, and 28 patients (35 percent) associations of microorganisms (2-3 microbes).

In the control group, from the tooth-gingival pockets (Fig 5), Staphylococcus aureus was found in 76.3 percent (in 29 of 38 people), Staphylococcus epidermidis - in 47.4 percent (in 18 of 38 people) and hemolytic streptococcus - in 29.0 percent (11 out of 38 people). In 9 out of 38 patients from the control group (in 23.7 percent) during the first examination, the causative agent of Candida was found in the periodontal pocket: Candida albicans and Candida tropicalis. In 22 people (in 57.9 percent) of 38 patients in the control group, microflora was detected in the form of a monoculture, and in 16 people (42.1 percent) - in the form of associations (2-3 microbes).

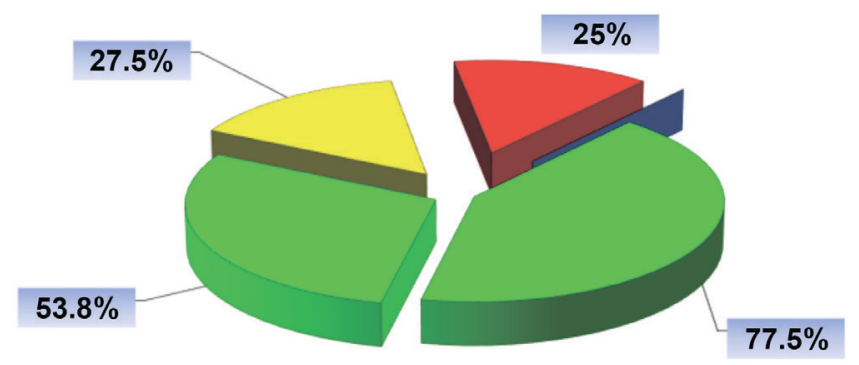

FIGURE 4. The proportion of certain types of microorganisms identified in the main observation group in patients with fractures of the alveolar processes of the maxilla and the mandible.

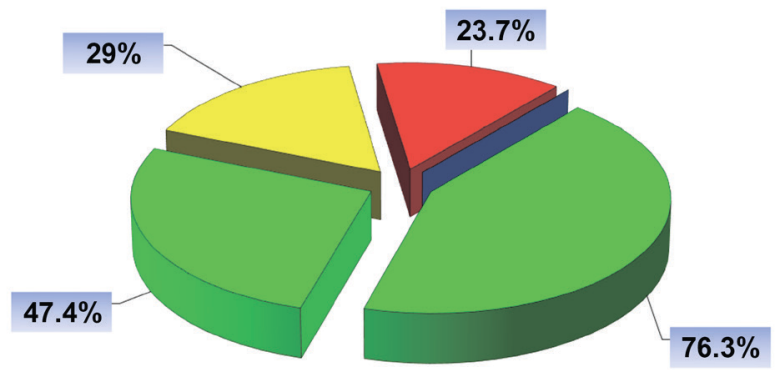

FIGURE 5. The proportion of certain types of microorganisms identified in the control group of observation in patients with fractures of the alveolar processes of the maxilla and the mandible. 
If we compare the species composition of the detected microflora depending on the examined group (main or control), then it should be noted that it was practically the same, ie did not differ significantly between the examined groups.

Monocultures of staphylococci showed sensitivity to aminoglycoside drugs and, to a lesser extent, to semisynthetic penicillins and anti-staphylococcal reserve antibiotics. Hemolytic streptococci showed sensitivity to most of the studied antibiotics, which did not depend on their associative links.

Before the removal of arch bars in the main group, Staphylococcus aureus was sown in 2.5 percent (in 2 out of 80 patients) from the periodontal pockets, Staphylococcus epidermidis and hemolytic streptococcus were not detected. Staphylococcus aureus was found as a monoculture. We did not find any fungal microflora after rinsing the mouth with the Tantum Verde.

In the control group of observation, after rinsing the mouth with traditional antiseptics in the periodontal pocket, Staphylococcus aureus was found in 23.7 percent (in 9 of 38 patients), Staphylococcus epidermidis - in 13.2 percent (in 5 of 38 people) and hemolytic streptococcus - in 15.8 percent (in 6 out of 38 people). In 4 out of 38 examined in the control group (10.5 percent), patients with fractures of the mandible in the periodontal pocket were re-detected the causative agent of Candida: Candida albicans and Candida tropicalis. Microorganisms were identified both as monocultures and in associative links with other microbes.

The Schiller-Pisarev test (ie, Svrakov iodine number) in patients with fractures of the alveolar processes of the maxilla and the mandible in the main observation group on the next day after reposition of fragments was $6.9 \pm 0.6$ points, which indicated the presence of an intense inflammatory process of the mucous membrane of the alveolar process, and in the control group $-6.8 \pm 0.7$ points (Fig 6). On the 7-8th day of the treatment in the patients of the main observation group, the Svrakov iodine number was $4.0 \pm 0.3$ points (moderately expressed inflammatory process in the mucous membrane of the alveolar process of the jaw), and in the control group - $5.7 \pm 0.4$ points (intense inflammation). On the 14-15th day, in patients with fractures of the alveolar processes of the jaws in the main group, the iodine number of Svrakov was $2.0 \pm 0.3$ points (mild inflammatory process in the mucous membrane of the alveolar process of the jaw), and in the control group $-4.7 \pm 0.5$ points

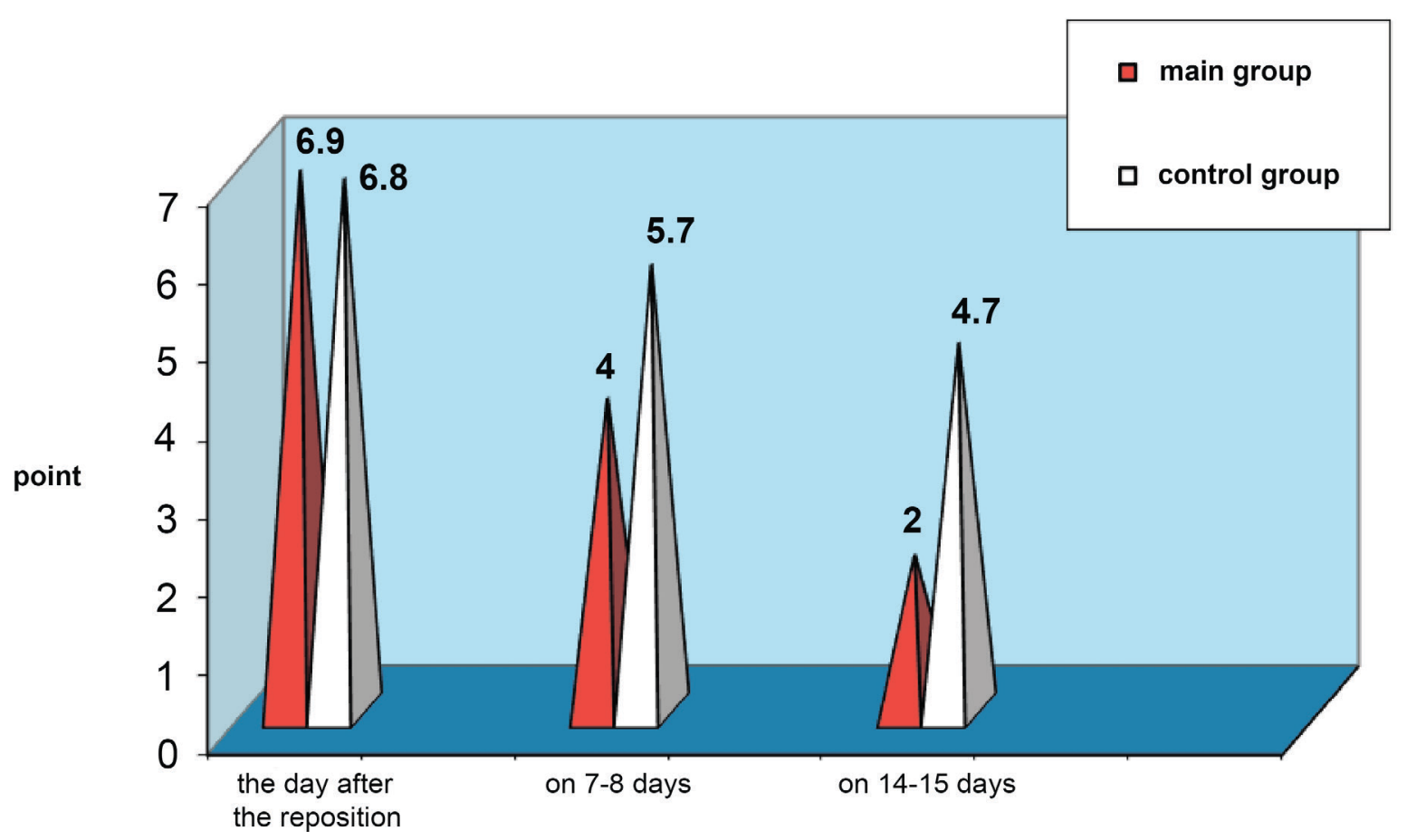

FIGURE 6. Schiller-Pisarev test (Svrakov iodine number) in patients with fractures of the alveolar processes of the jaw in the dynamics of treatment. 
(moderately expressed inflammatory process in the mucous membrane of the alveolar process). The Schiller-Pisarev test in patients with fractures of the alveolar process of the jaws in the dynamics of the treatment indicated a high anti-inflammatory efficacy of the drug "Tantum Verde."

The index of gingivitis in patients with fractures of the alveolar processes of the jaws in the main observation group on the next day after reposition of fragments was $1.32 \pm 0.11$ points, in the control group - $1.33 \pm 0.09$ points, which indicated the presence of an average degree of gingivitis (Fig 7). On the 7-8th day of the treatment, the gingivitis index in patients of the main group was $0.81 \pm 0.07$ points (mild gingivitis), and in the control group $1.42 \pm 0.11$ points (moderate gingivitis). On the 1415th day of treatment in patients with fractures of the alveolar processes of the jaws, the gingivitis index in the main observation group was $0.61 \pm 0.05$ points (mild gingivitis), in the control group - $1.35 \pm 0.11$ points (moderate gingivitis). The gingivitis index indicated the high efficacy of "Tantum Verde."

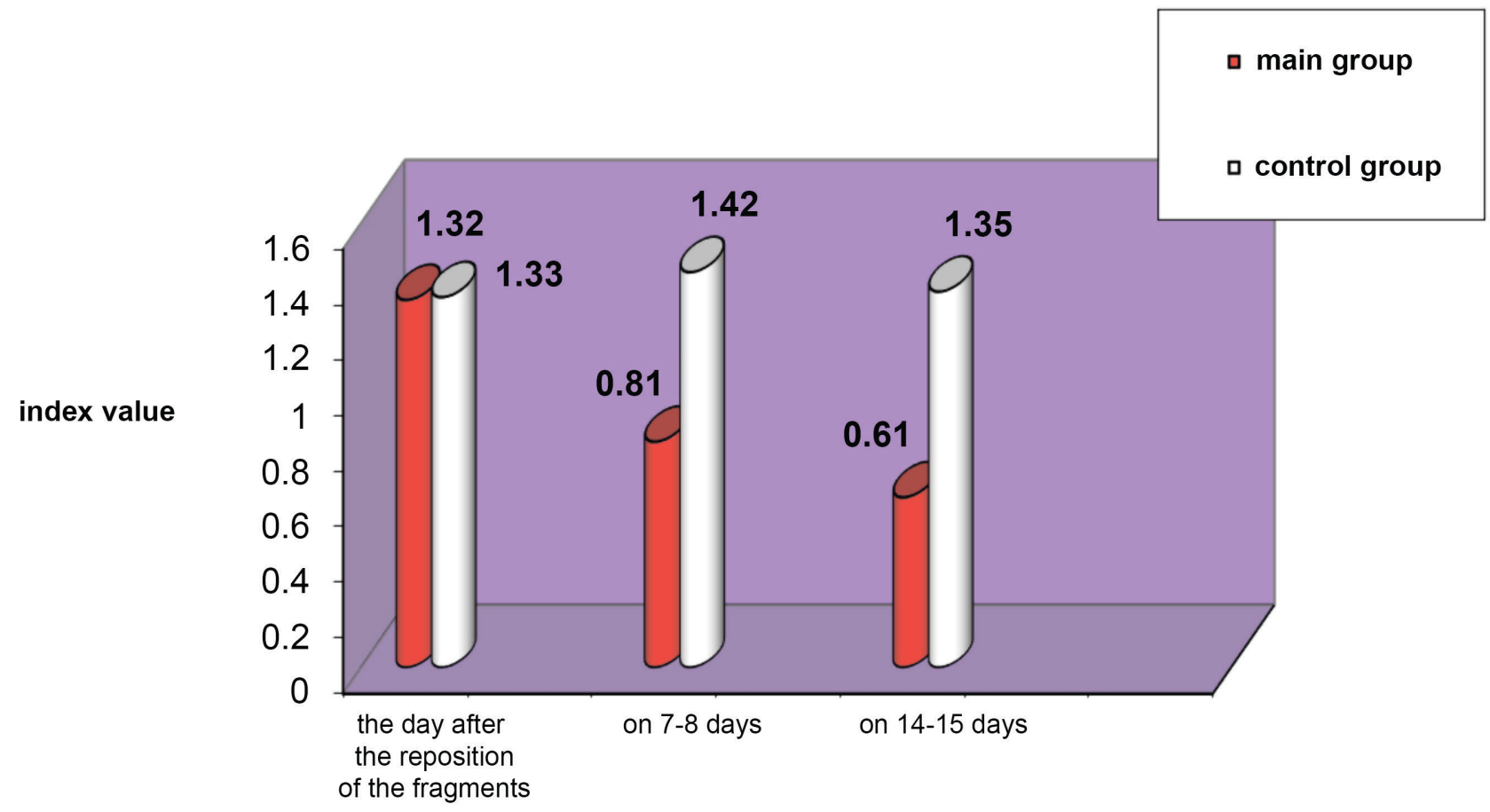

FIGURE 7. Changes in the gingivitis index in patients with fractures of the alveolar processes of the jaws in the dynamics of treatment.

Bad breath in patients with fractures of the alveolar processes of the jaws in the main and control groups of observation (Fig 8) on the next day after the reposition of fragments was recorded in 100 percent of cases. After 7-8 days of hygienic treatment of the oral cavity, in the main observation group, an unpleasant odor was detected in 14 out of 89 subjects (15.7 percent), and in the control group - in 21 out of 40 patients (52.5 percent). After 14-15 days of hygienic treatment of the oral cavity, an unpleasant odor in the main group persisted in 2 out of 89 patients ( 2.3 percent), and in the control group - in 17 out of 40 people ( 42.5 percent). The examinations indicated a high $(p<0.001)$ deodorizing effect of the drug "Tantum Verde" in comparison with the control group of the examination.

We performed thermometric examinations of 129 patients with fractures of the jaws (89 people - the main group, 40 people - the control group). Thermal asymmetry was determined on the mucous membrane of the alveolar process of the jaws in the area of the fracture gap and the obtained temperature was compared with the symmetrical area on the intact side. The data obtained while examination are presented in Table 1. It was revealed that on the mucous membrane of the alveolar process at the 


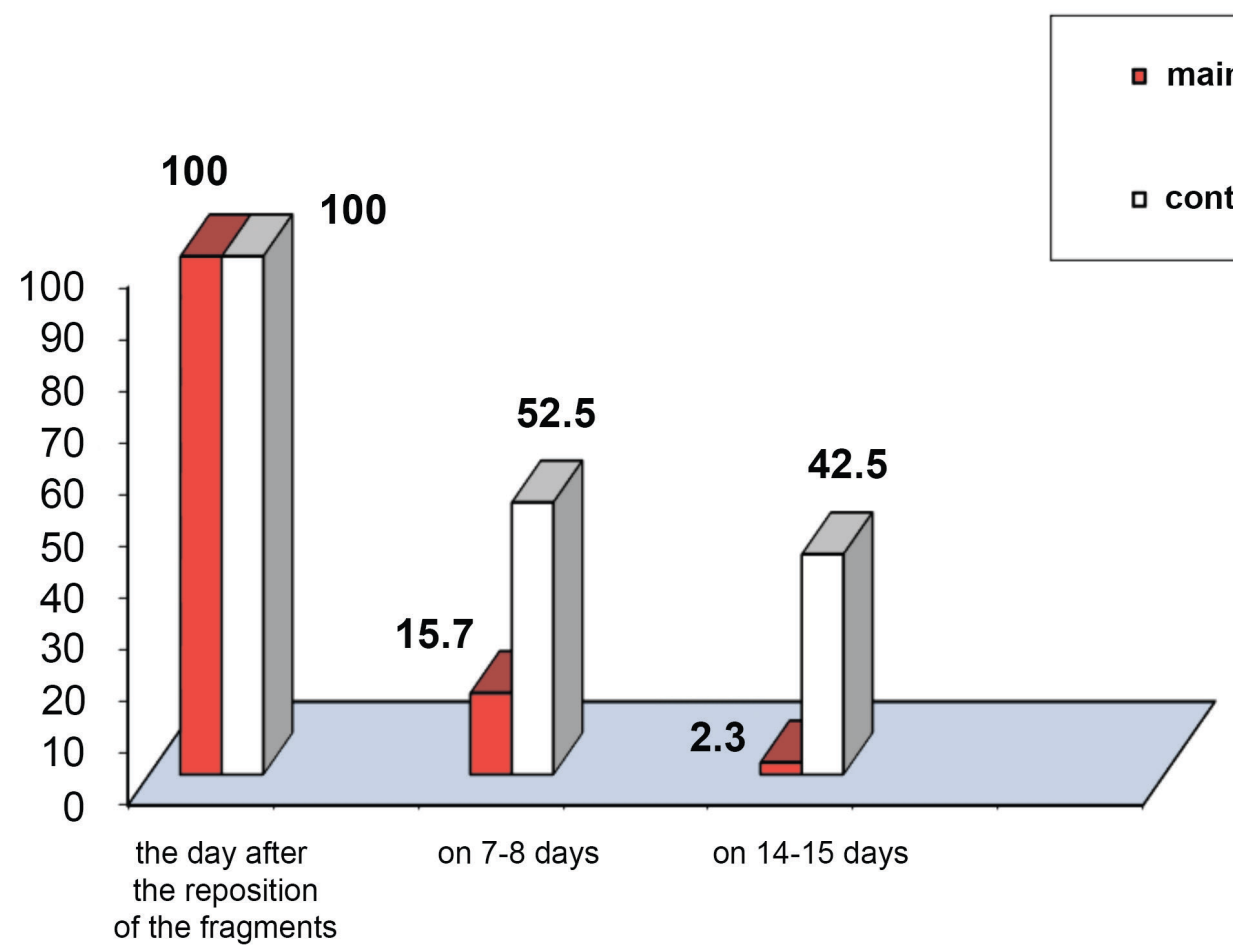

FIGURE 8. Frequency of occurrence of bad breath in patients with fractures of the alveolar processes of the jaws in the dynamics of the examination.

site of injury to the mandibular bone, on the first day during treatment there was a significant $(<$ $0.01)$ increase in local temperature in all examined patients up to $1.7 \pm 0.2^{\circ} \mathrm{C}$ (main group) and 1.8 $\pm 0.2^{\circ} \mathrm{C}$ (control group). On the 7 th day of the treatment, the thermal asymmetry of the mucous membrane of the alveolar process was: $1.1 \pm 0.3^{\circ} \mathrm{C}$ (main group) and $1.2 \pm 0.3^{\circ} \mathrm{C}$ (control group). On the 14th day of the treatment, the thermal asymmetry of the mucous membrane of the alveolar process in the area of the fracture site in the main observation group returned to normal (Table 1). In the control group of observation, the normalization of thermometric indicators occurs only on the 18 20th day. The examinations indicated a high $(p<$ $0.001)$ anti-inflammatory effect of the drug "Tantum Verde" in comparison with the control group of patients.

The soft tissues around the jaws on the side of the injury, the day after the reposition of the jaw fragments,

TABLE 1. Thermal Asymmetry Indicators of the Mucous Membrane of the Alveolar Process of the Jaws in Patients of the Main and Control Groups.

\begin{tabular}{|c|c|c|c|c|}
\hline \multirow{2}{*}{ Observation Group } & \multirow{2}{*}{ Number of Examined } & \multirow{2}{*}{ Time of Examination } & \multicolumn{2}{|c|}{$\Delta \mathrm{T}-$ Thermal Asymmetry (in $\left.{ }^{\circ} \mathrm{C}\right)$} \\
\hline & & & $\mathbf{M} \pm \mathbf{m}$ & $p$ \\
\hline \multirow{3}{*}{ Main group } & \multirow{3}{*}{89} & While hospitalization & $1.7 \pm 0.2$ & $<0.001$ \\
\hline & & On the 7th day & $1.1 \pm 0.3$ & $<0.05$ \\
\hline & & On the 14th day & $0.5 \pm 0.2$ & $>0.05$ \\
\hline \multirow{4}{*}{ Control group } & \multirow{4}{*}{40} & While hospitalization & $1.8 \pm 0.2$ & $<0.001$ \\
\hline & & On the 7th day & $1.2 \pm 0.3$ & $<0.05$ \\
\hline & & On the 14th day & $1.2 \pm 0.2$ & $<0.05$ \\
\hline & & On 18-20 days & $0.9 \pm 0.3$ & $>0.05$ \\
\hline Healthy persons & 33 & & $0.5 \pm 0.1$ & \\
\hline
\end{tabular}

Note: $p$ - significance of differences in comparison with healthy people. 
were edematous (with the presence of hemorrhages) and infiltrated (Fig 9) in all patients (100 percent) in both the main and control groups. In the main group, moderate edema and infiltration of the soft tissues were observed in 49 of 89 patients (55.1 percent), and insignificant - in 40 people (in 44.9 percent). In the control group: moderate edema and infiltration - in 21 out of 40 people (in 52.5 percent), and insignificant - in 19 people (in 47.5 percent). 2-3 days after the reposition of the fragments in the main observation group, moderate edema and infiltration of the soft tissues was observed in 24 out of 89 patients (in 27.0 percent), and insignificant in 65 patients (in 73 percent). In the control group: moderate edema and infiltration - in 20 out of 40 people (in 50 percent), and insignificant - in 20 people (in 50 percent). In 5-6 days after the fragments reposition in the main group, edema and infiltration of the soft tissues around the jaws in all patients (in 100 percent) were insignificant. In the control group: moderate edema persisted in 7 out of 40 people (in 17.5 percent), and insignificant - in 33 people (in 82.5 percent). The examinations indicated a high $(p<0.001)$ anti-inflammatory effect of the drug "Tantum Verde" in comparison with the control group of the examined.

The next day after reposition, post-traumatic ruptures of the mucous membrane covered with dirty gray plaque of the alveolar process (Fig 10), were in all patients (100 percent) in a moderate amount in both: the main and control groups. In the main observation group, a moderate amount of plaque on the mucous membrane in the fracture gap in 2-3 days after reposition was in 20 of 89 people (in 22.5 percent), and insignificant - in 69 people (in 77.5 percent). In the control group: moderate - in 31 out of 40 people (in 77.5 percent), and insignificant - in 9 people (in 22.5 percent), and insignificant - in 69 people (in 77.5 percent). In the control group: moderate - in 31 out of 40 people (in 77.5 percent), and insignificant - in 9 people (22.5 percent). 5-6 days after reposition in the main group, they were insignificant in all patients (100 percent). In the control group: moderate plaque remained in 4 out of 40 people (10.0 percent), and insignificant - in 36 people (90.0 percent). The examinations indicated a high $(p<0.001)$ antiinflammatory effect of the "Tantum Verde" drug in comparison with the control group of the examined.

When treating patients with fractures of the alveolar processes of the jaws in the main observation group, moderate pain in the area of the pathological focus (Fig 11) was detected in 57 out of 89 examined (64.1 percent), and insignificant in 32 people (35.9

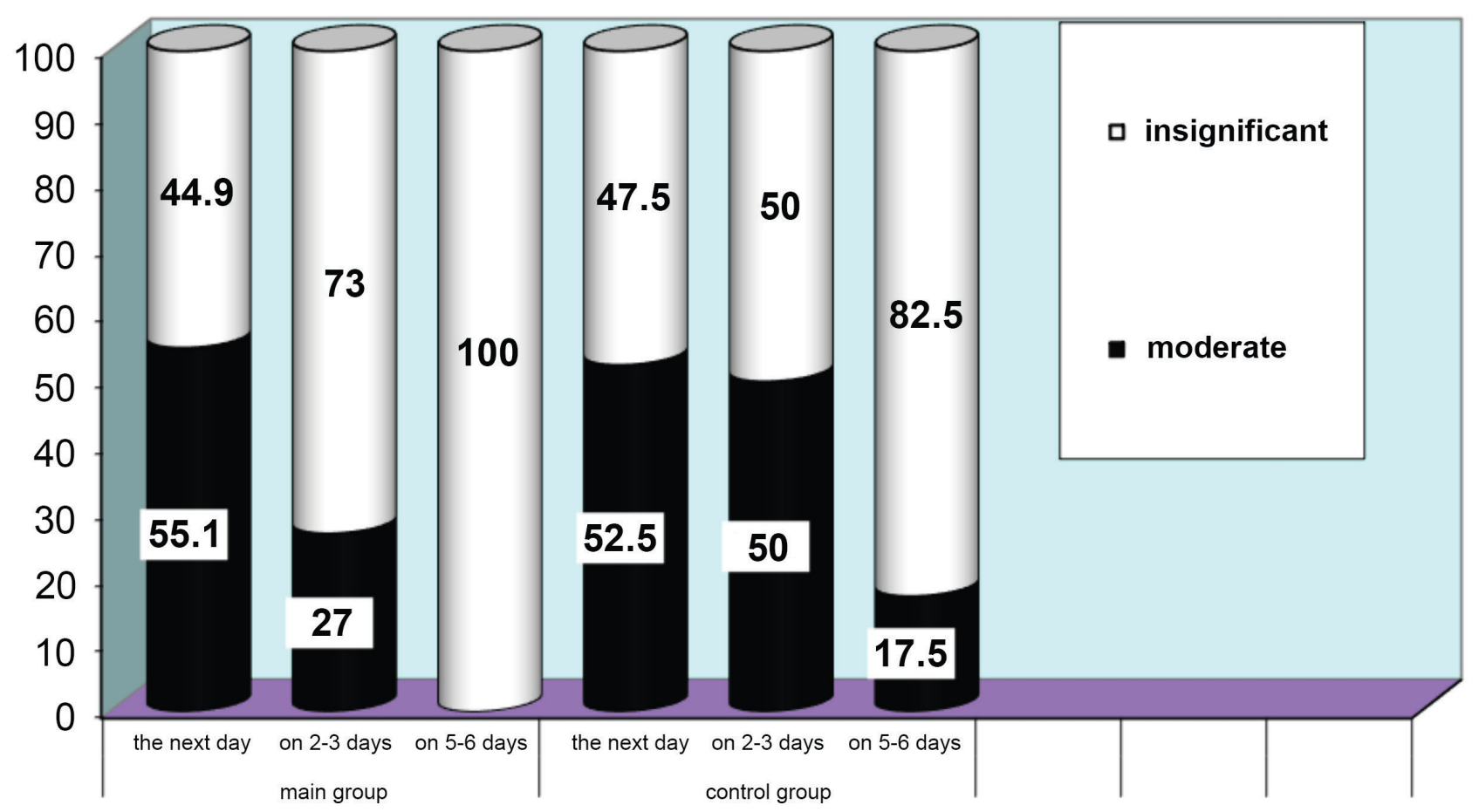

FIGURE 9. Changes in edema and soft tissues infiltration in the area of jaws in the treatment dynamics of patients after reposition of jaw fragments. 


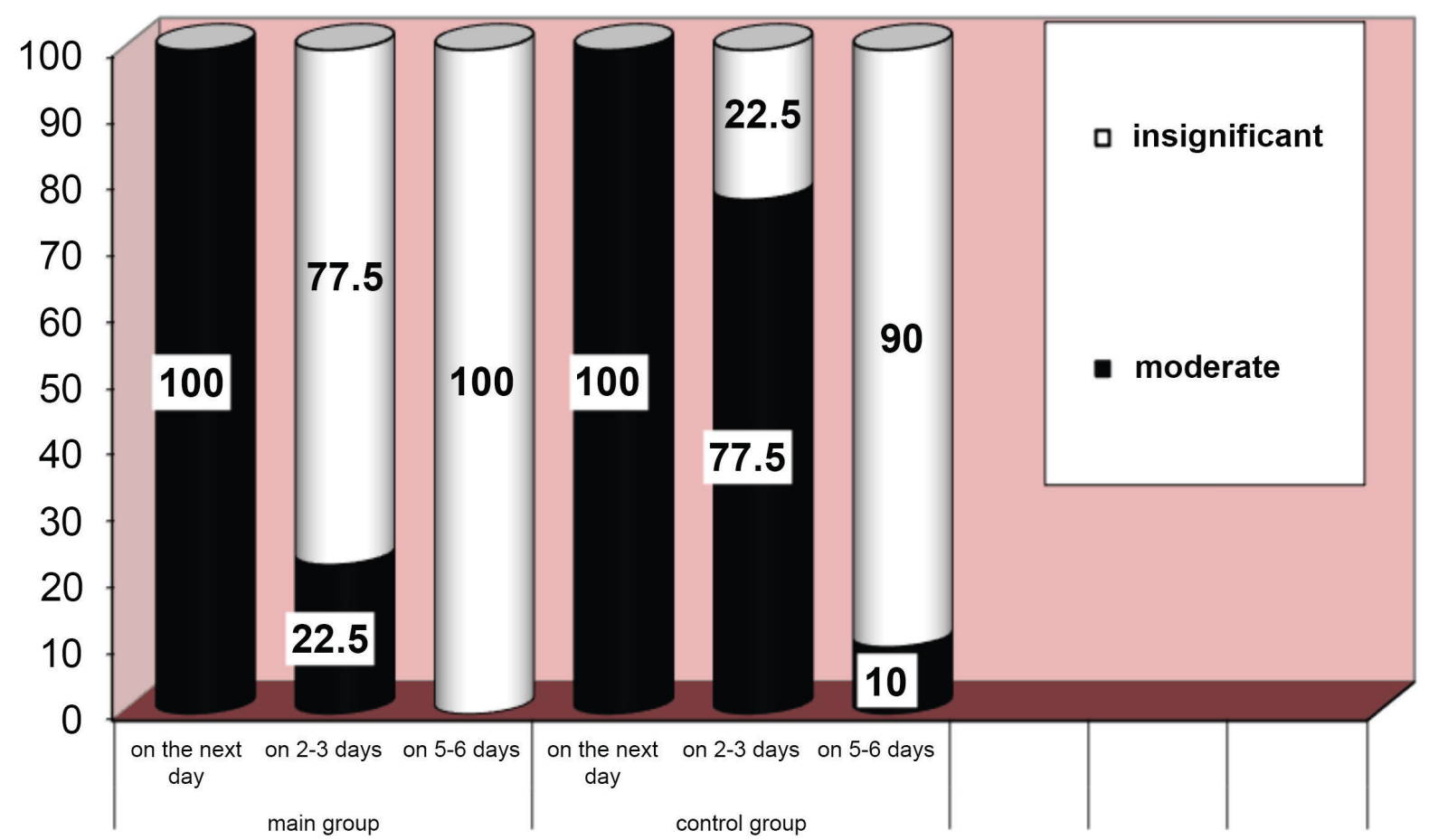

FIGURE 10. Changes of plaque on a mucous membrane in a fracture crack in treatment dynamics of patients with a fracture of alveolar processes of jaws.

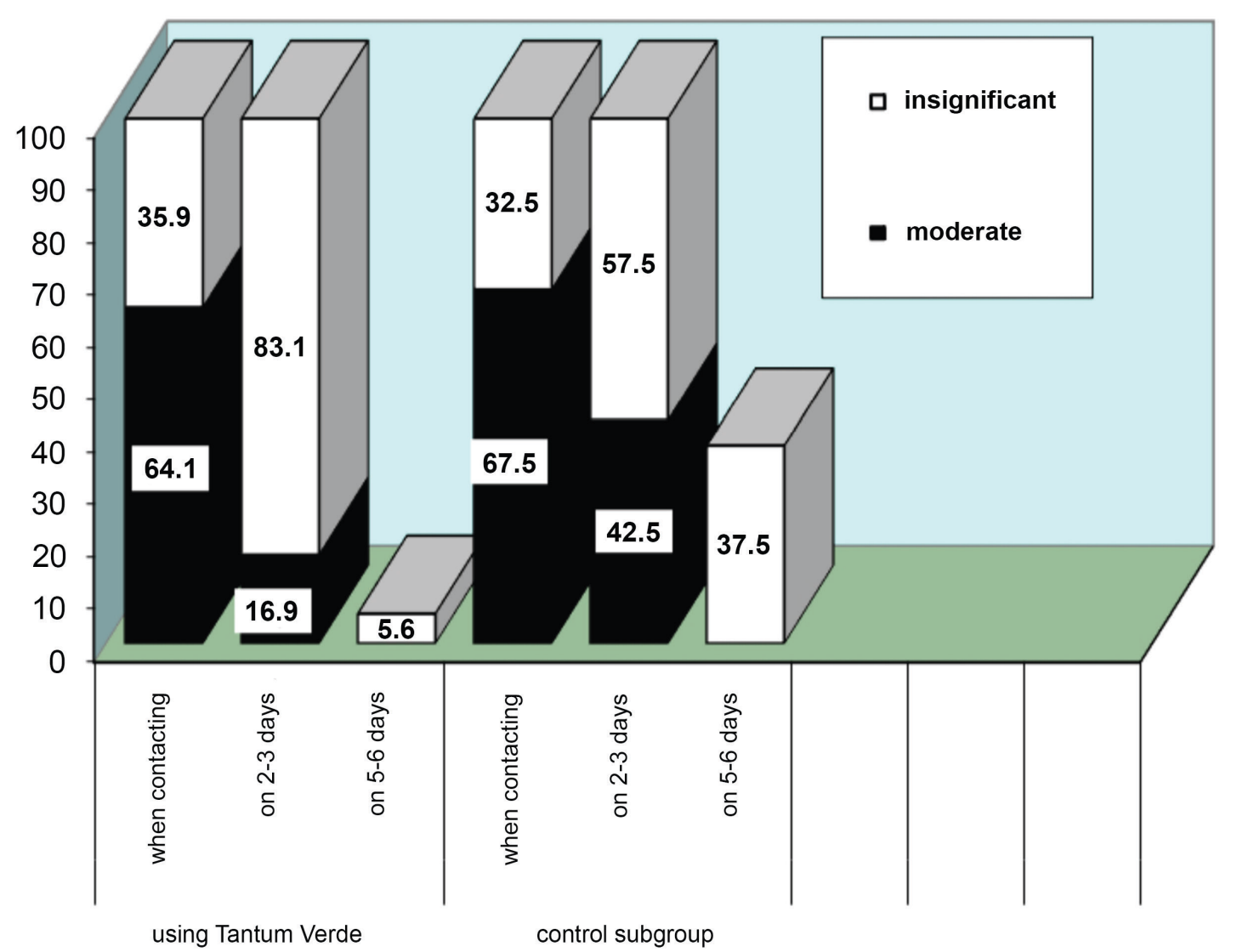

FIGURE 11. Changes of plaque on a mucous membrane in a fracture crack in treatment dynamics of patients with a fracture of alveolar processes of jaws. 
percent). In the control group of patients, moderate pain was noted in 27 out of 40 examined (67.5 percent), insignificant - in 13 people (32.5 percent). After 2-3 days of treatment, the patients of the main group had moderate pain in 15 of 89 examined (16.9 percent), and insignificant pain in 74 people (83.1 percent). In the control group, during the same period, moderate pain was recorded in 17 out of 40 patients (42.5 percent), and insignificant pain in 23 people (57.5 percent). After 5-6 days from the start of treatment, the patients of the main group had no pain of a moderate nature, and 5 out of 89 examined (5.6 percent) had insignificant pain, the rest of the examined did not have pain at all. The patients in the control group also did not have moderate pain, and 15 out of 40 examined patients ( 37.5 percent) had insignificant pain, and the rest of the patients in the same group had no pain symptoms. The examinations indicated a high $(p<0.001)$ local anesthetic effect of the "Tantum Verde" drug in comparison with the control group of examined (Fig 11).

Early inflammatory complications of patients with fractures of the alveolar processes of the maxilla and mandible in the main observation group in the form of gingivitis were observed in 2 of 89 examined (2.3 percent). In the control observation group we detected early inflammatory complications in the form of gingivitis in 9 out of 40 examined (22.5 percent).

The use of the proposed method of hygienic treatment with the "Tantum Verde" of the oral cavity for patients with fractures of the alveolar processes of the jaws made it possible to reduce the time of consolidation of fragments for $2.4 \pm 0.7$ days.

\section{CONCLUSIONS}

It has been established that the nonsteroidal anti-inflammatory drug "Tantum Verde" has an expressed antibacterial, anti-inflammatory and deodorizing effect, and also provides a good cleaning of metal structures located in the vestibule of the oral cavity (arch bars). On the basis of the conducted examinations, it was proved that the anti-inflammatory and analgesic efficiency of the "Tantum Verde" drug in the treatment of patients with fractures of the alveolar processes of the maxilla and mandible significantly exceeds traditional methods of treatment. The use of the proposed method of hygienic oral cavity treatment for patients with fractures of the alveolar processes of the jaws allowed to significantly reducing the number of early inflammatory complications and the time of consolidation of alveolar fragments of the jaw.

\section{AUTHOR CONTRIBUTION}

Conceptualization: Tymofieiev OO. Data and interpretation acquisition: Ripa VM, Myroshnyk AO, Savytskyi OO, Dubichenko SI, Blinova VP, Uharska OA, Serga OO. Drafting of the manuscript: Maksymcha SV, Yarifa MO. Critical revision of the manuscript: Tymofieiev OO, Ushko NO, Fesenko II. Approval of the final version of the manuscript: all authors.

\section{REFERENCES}

1. Lauridsen E, Gerds T, Andreasen JO. Alveolar process fractures in the permanent dentition. Part 2. The risk of healing complications in teeth involved in an alveolar process fracture. Dent Traumatol 2016;32(2):128-39. https://doi.org/10.1111/edt.12229

2. Tymofieiev OO. Maxillofacial surgery and surgical dentistry [Russian]. Volume 1. Kyiv, Ukraine: Medicine; 2020

3. Gutmacher Z, Peled E, Norman D, Lin S. Alveolar bone fracture: pathognomonic sign for clinical diagnosis. Open Dent J 2017;11:8-14. https://doi.org/10.2174/1874210601711010008

4. Lim L, Sirichai P. Bone fractures: assessment and management. Aust Dent J 2016;61 Suppl 1:74-81. https://doi.org/10.1111/adj.12399

5. Tymofieiev OO, Fesenko II, Tymofieiev OO. Condition of the teeth in fracture gap of the mandible. J Diagn Treat Oral Maxillofac Pathol 2017;1(1):41-53. https://doi.org/10.23999/j.dtomp.2017.1.6

6. Instructions for medical application of TANTUM VERDE $^{\circledast}$ (TANTUM VERDE ${ }^{\circledast}$ ). Order of the Ministry of Healthcare of Ukraine No. 1789 dated 08/04/2020 